\title{
MHD Reconnection Model for Optical Jets, H-H Objects and GGD Objects
}

\author{
Hitoshi HANAMI ${ }^{\star}$ and T. TAJIMA \\ Department of Physics, University of Texas at Austin, \\ Austin, TX78712-1060, U.S.A.
}

Many objects have been observed that are excited by shocks, but nevertheless do not form a shell shape in a variety of astrophysical environments such as in protostellar and circumstellar medium or as seen in optical jets, $\mathrm{H}-\mathrm{H}$ objects, and GGD objects. The models for these objects, which have been proposed for explaining observed spectrum, can be divided to two types. First one is the cloudlets interaction model (e.g., Schwartz 1978; Norman and Silk 1979; Raga, Böhm, and Solf 1986). Second one is the radiative jet model in which the knotty structure associated in some $\mathrm{H}-\mathrm{H}$ objects can be suggested as the shock of Mach disks in "underexpanded" jets (e.g., Mundt 1985; Hartigan 1989). However, the wiggles and the irregular knots have been observed in some $\mathrm{H}-\mathrm{H}$ objects (e.g., knots; HH33, HH40, and wiggles; HH12, HH7-11), which could not be explained well by the two models above. On the other hand, from the observations for maser emissions in star forming regions, the strong magnetic field is inferred(e.g., Fiebig and Gusten 1989). The magnetic field seems to be important for the dynamical motion in star forming regions. Then, a magnetohydrodynamical reconnection model for these phenomena is investigated. We consider the interaction between dense magnetized cloudlet and wind from the central star, through an 2-D MHD code with the MacCormack-Doner cell hybrid scheme to well represent shock phenomena as well as advective phenomena. The density contours and magnetic field lines of numerical results for $\gamma=5 / 3$ and 1.1 are shown in figure (a) and (b), respectively. We present two case for $\gamma=5 / 3$ and 1.1. Results show that the compression effects related to the adiabatic indexes $\gamma$ are important in the shocked region. The interaction between the cloudlet and wind induces the bow shock around the cloudlet and elongated magnetic structure in the downstream direction, which is similar between two cases. However, the downstream flow patterns behind the cloudlet are different for each other. For $\gamma=1.1$, we have a jet-like structure in downstream region. This is induced by magnetic reconnection with strong compressed effects which produces secondary plasma acceleration. This magnetic reconnection is fast (Patscheck) type, which have been well studied for the model of the earth magnetospheric substorm, related to the slow shock formation and the strong plasma jet generation (e.g. Sato and Hayashi 1979). Furthermore, these behavior is similar

* Present Address; Department of Physics, Kyoto University, Kyoto, 606, JAPAN 
to the disconnection events of comets (Alfvén 1957, Niedner 1980), which suggests us the knots in $\mathrm{HH}$ objects can be explained as some separated reconnection points in which the magnetic energy is released explosively. For real interstellar medium, $\gamma=5 / 3$, however, we must consider the effect of radiative cooling (Spitzer 1978). This cooling seems to be important for outflows in the star forming regions (Hanami and Sakashita, 1987) Then, we can interpret the compression effect with small $\gamma$ as that of the cooling, approximately. This compression mechanism related to the cooling effect in interstellar and magnetic reconnections are important for interpreting the formation, and the structure, and the dynamics of optical jets, $\mathrm{H}-\mathrm{H}$ objects, and GGD objects.

Figure

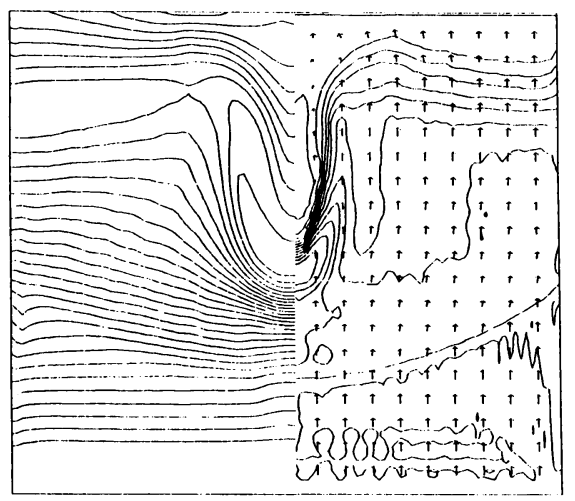

(a)

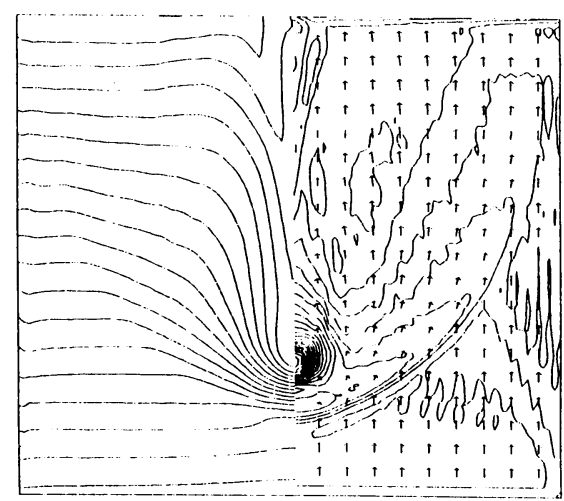

(b)

\section{References}

Alfvén, H. 1957, Tellus 9:92.

Fiebig, D. and Güsten R., 1989, A.Ap., 333, 1989.

Hanami, H. and Sakashita, S., 1987, in Proceedings of IAU symposium No.115 "Star Forming Regions", ed. M.Peimbert and J.Jugaku (D.Reidel Publ. Co.), P.391.

Hartigan, P. 1989, Ap.J., 339, 987.

Mundt, R., 1985, in Protostars and Planets II, ed. D.C.Black and M.S. Matthews (Tucson: University of Arizona Press), P.414.

Niedner, M.B. 1980, Ap.J., 241, 820.

Norman, C., and Silk, J. 1979, Ap.J., 228, 197.

Raga, A. C., Böhm, K. H., and Solf, J. 1986, A.J., 92,119.

Sato, T. and Hayashi, T., 1979, Phys. Fluids, 22, 1189.

Schwartz, R.D., 1978, Ap.J., 223, 884.

Spitzer,L.Jr. 1978, Physical Processes in the Interstellar Medium (John Wiley \& Sons, Inc. ). 